\title{
Long-Term Results (Seven and More Years) of Sparing Treatment of Subjects with Descending Aortic Aneurysms
}

\section{Vladimir Petrovich Krylov1,2, Dmitry Evgeniyevich Shevtsov², Gennadiy Adolfovich Popel1, Valentina Nikolaevna Gayduk ${ }^{1,2}$, Leonid Ivanovich Reut ${ }^{1}$, Aleksey Leonidovich Smaliakou', Nadezhda Vladimirovna Mankevich ${ }^{1,2}$}

\author{
${ }^{1}$ Republican Research and Practical Centre of Cardiology, Minsk, Belarus \\ ${ }^{2}$ Belarusian Association of Physicians Ministry of Health of Belarus, Minsk, Belarus \\ Email: krylov_v_p@mail.ru, a.mrochek@mail.by, leotit310@gmail.com
}

How to cite this paper: Krylov, V.P., Shevtsov, D.E., Popel, G.A., Gayduk, V.N., Reut, L.I., Smaliakou, A.L. and Mankevich, N.V. (2019) Long-Term Results (Seven and More Years) of Sparing Treatment of Subjects with Descending Aortic Aneurysms. Health, 11, 661-670.

https://doi.org/10.4236/health.2019.116055

Received: March 26, 2019

Accepted: June 3, 2019

Published: June 6, 2019

Copyright $\odot 2019$ by author(s) and Scientific Research Publishing Inc. This work is licensed under the Creative Commons Attribution International License (CC BY 4.0).

http://creativecommons.org/licenses/by/4.0/

\begin{abstract}
Descending aortic aneurysms (DAAs), including dissecting aneurysms (DA) have a multifactorial etiology and pathogenesis, therefore raising questions about the leading role of operative treatment to repair the lesion. Objective: To investigate remote ( 7 and more years) results of treatment in patients with DAAs in operative treatment only if there is a danger of aneurysm rupture. A total of 82 patients with atherosclerotic DAA identified between 2008 and 2011, and 22 patients with type 1 or 3 DeBakey dissecting aneurysms (DA) who had not been operated in the acute period due to a number of reasons were examined. The follow-up period of these groups was 7 or more years. When using a sparing treatment to treat DAA, we saw survival of 90.1 at 2 years, $76.8 \%$ at 4 years, $59.4 \%$ at 6 years, and $57.5 \%$ at 7 years with the uniform increase in mortality rate, mainly due to a comorbidity. Survival in DA group $(77.3 \%)$ was better due to a younger age and was $68.4 \%$ in operated and $54 \%$ in non-operated patients at 7 years. It is symptomatic that the aneurysm rupture rate was not always affected by operative treatment. Therefore, it seems like medical treatment is more consistent with etiopathogenesis of the disease compared to surgery during the stabilization period.
\end{abstract}

\section{Keywords}

Descending Aortic Aneurysms, Medical and Surgical Treatment, 7-Year

Treatment Results, Survival

\section{Introduction}

In this study, assuming a common nature of underlying pathogenetic processes 
occurring in the aortic wall as a result of atherosclerotic lesion, we decided to combine the terminology of the descending thoracic and abdominal aortic segments into "descending aortic segments", and used the term "descending aortic aneurysms".

Among the cases with DAAs, abdominal aortic aneurysms (AAAs) account for $75 \%$, while it's just $25 \%$ for descending thoracic aneurysms (DTAs). Due to population aging, high rates of arterial hypertension and improved diagnosis, the detection rate has increased threefold for AAA, and 10 times for minor AAAs [1] in the last 3 decades. After age 60 , AAA is diagnosed in $6 \%$ of males, and $1 \%$ of females [2].

A combination of disgenias and degenerative and inflammatory changes in the aortic wall is the underlying cause of aneurysm formation. Moreover, the risk factors for DAA include old age (from 60 years old), male sex, smoking, genetic susceptibility, arterial hypertension, and hypercholesterolemia [3]. The aortic rupture, a severe complication of AAA is associated with a diameter of the aorta, the rate of its expansion $(0.5 \mathrm{~cm}$ at 6 months), current smoking status, and persistent blood pressure (BP) [3].

A family history of aneurysm increases fourfold the risk of AAA [4]. In this case, it might have something to do with connective tissue dysplasia poorly differentiated, in the same way as with the ascending aortic aneurysm observed in young patients with Marfan's syndrome whose genetic predisposition has been confirmed and is estimated by fibrillin- 1 and matrix metalloprotease- 9 deficiency during the embryonic stage of gestation [5].

Oxidative stress and chronic inflammation are the major mechanisms of degenerative processes associated with vascular aging [6]. The key role in the development of those factors belongs to a permanent hyperactivation of renin-angiotensin-aldosterone system, which is manifested through the active hormone angiotensin-II (ATII). A pro-inflammatory effect of AT-II implies the activation of cytokines including tumor necrosis factor-alpha and interleukins [7], further stimulating a local production of C-reactive protein (CRP) which increases vascular inflammation and endothelial dysfunction even more [8].

AT II is crucial for the destruction of aortic media activating matrix metalloprotease-2 which promotes elastin thinning, flattening and fragmentation. Moreover, it enhances collagen production in the aortic wall, intimal thickening, and increased arterial stiffness [9]. The increased density of elastic arteries increases pulse wave velocity (PWV) and makes it earlier return as a reflected wave [10].

There is much debate in literature sources concerning treatment and management options for these patients. Many authors think that the surgical approach is a mainstay of therapy to treat the disease. The surgery can be performed when the diameter of the aneurysm is more than $5.5 \mathrm{~cm}$. Although the surgical methods have been proven effective, not all patients can safely tolerate the operation. 
A large-scale, population-based investigation (DECREASE-1) [11] showed that the risk of intraoperative cardiac complications in subjects with abdominal aortic aneurysm having 5 or more risk factors out of 7 (including previous myocardial infarction, effort angina pectoris, chronic heart failure, type 2 diabetes mellitus, chronic kidney disease, a history of stroke, and the age older than 70) was $32 \%$.

Two randomized investigations [12] assessed the feasibility of surgery to treat AAA with the diameter of $<5.5 \mathrm{~cm}$, and showed that the survival of patients had been unaffected by either aneurysm diameter, or delayed Echo- and CT-guided surgery at $4.6-4.8$ years. Moreover, the delay was not meaningful in the immediate and interim periods after surgery [1].

Endovascular methods are an alternative to a conventional surgical modality. Transluminal stent-graft implantation into a damaged part of the aorta is less traumatic, but easy to accomplish in only one-third of all cases with dissecting aneurysms due to a poor proximal aortic segment, functioning distal fenestration, visceral and renal perfusion severely deviated and significantly abnormal.

There is a third treatment option that includes the follow-up and conservative treatment of large patient cohort who has been refused the operation due to a comorbidity, older age or small size of AAA. The aim of this option is to prevent aneurysm progression and prolong patients' life expectancy. Advocates of this option suggest that it should be used in all patients with DAAs before and after operation for optimal surgical results.

Unfortunately, no works can be found, at least in Russian editions, that would describe conservative treatments including sparing to treat aortic aneurysms. In our work, we relied on data obtained from EVAR investigations and Japanese data. As far as we know, the surgical treatment modality is the key approach worldwide to treat aneurysms. At the same time, non-operated patients are frequently treated by therapeutists who might not always have clear guidelines in place to strictly follow.

Objective: To investigate remote ( 7 and more years) results of treatment in patients with DAAs in operative treatment only if there is a danger of aneurysm rupture.

\section{Materials and Methods}

\section{Patient Characteristics}

A total of 82 subjects with atherosclerotic DAA identified in the period between 2008 and 2011 were examined. Additionally, we analyzed the results of this treatment approach over the same follow-up period in 22 individuals with dissecting aneurysm (DA) who had not been operated during the acute period for a variety of reasons. Thus, the follow-up period of the survivors lasted for 7 years and more. Patients with DAs were categorized as type 1 and type 3 by DeBakey classification.

Patients with terminal organ dysfunction and chronic purulent infection older 
than 85 years old were not included in the investigations.

The key comparison parameters were aneurysm expansion dynamics; the presence/progression and potential correction of comorbidity; compliance to treatment, risks of aneurysm rupture (pain syndrome or aneurysm diameter abruptly increased, CRP) and potential of operative treatment secondary to medical therapy.

\section{Methods}

1) Examination Methods: Clinical, heart US, US of brachiocephalic arteries and abdominal organs with the estimation of the descending aortic diameter; US of the lower extremity arteries, CT and CT-angiography, blood lipid profile, and CRP test.

2) Methods of Surgical Treatment: Aortoiliac/femoral reconstruction or stent-graft implantation.

3) Methods of Conservative Treatment: Based on data obtained from literature sources as well as our own expertise, we opted to use a multifaceted medical therapy with the following treatments:

a) Beta-blockers to slow down the progression of aneurysm based on data mentioned by Crawford back in 1984 about delayed progression of aortic aneurysms in patients with Marfan's syndrome when using beta-blockers compared to those previously untreated with beta-blockers [13]; to reduce risks of aortic rupture through the effects produced on elastin, collagen, and sympathoadrenal system responsible for collagen production [14]; to reduce susceptibility of beta-receptors to emotional stressors when biopolymers of aortic tissue get afflicted [15].

b) ACE-inhibitors to reduce the size of atherosclerotic plaque based on their anti-apoptotic, anti-inflammatory [16] and endothelium-stabilizing effects [17].

c) Dihydropyridine calcium-channel blockers to reduce central aortal BP, nocturnal BP and variability of BP [18] [19], as well as aortic stiffness and the detrimental effect produced thereon by the reflected wave [17].

d) Macrolides (azithromycin) to prevent the growth of aneurysm affecting the inflammatory processes within the aorta with CRP more than $3 \mathrm{mg} / \mathrm{L}$ [20]; and based on the evidence of their non-microbial effect and inhibition of the synthesis and secretion of pro-inflammatory cytokines [21].

e) Long-term use of statins to reduce cholesterol and LDLP levels; to improve endothelial function and prevent vasospasm producing anti-inflammatory and antioxidant effects [22] [23] [24] diminishing the size and calcification of atherosclerotic plaque [24].

f) Antiplatelet agents [25].

Statistical process methods: Student's t-test to assess the reliability of the findings.

\section{Results}

The results of the sparing treatment obtained from 104 subjects with newly di- 
agnosed descending aortic aneurysm seven or more years ago have been assessed. Of them, 82 had atherosclerotic lesion, and 22 had aortic dissection with no significant atherosclerosis. Those patients were distributed into two subgroups: Operated with medical treatment before and after surgery, and patients treated conventionally (Table 1 ). In patients with atherosclerosis, the mean age was 64.1 years old in operated, and 74.4 years old in non-operated. In individuals with dissection, the mean age was 58.3 years old in operated and 69.2 years old in non-operated.

Due to a large age variation observed in our groups and subgroups, no statistical differences between them were identified. However, the mean age of individuals with aortic dissection was smaller compared to those with atherosclerotic lesions, and it was significantly smaller in operated compared to those treated conventionally. It drew a distinction in comorbidity and consequent mortality between operated and treated conventionally.

The seven-year mortality rate of sparing treatment was nearly twice as high among patients with atherosclerotic lesion compared to those with DA $(43.9 \%$ vs. $22.7 \%$, respectively). Additionally, a comorbidity-related mortality rate among DAA and DA patients was $60 \%$ in each group. The different values of mortality rate are due to a great proportion of aortic ruptures due to DAA among both operated and non-operated, and fatal outcomes as a result of surgery.

Thus, a comorbid condition was a key cause in the structure of mortality. Still, only $16.7 \%$ of operated DAA patients died of it while among non-operated, it was $69 \%$. There were no DA patients who died of a comorbid condition. In contrast, $75 \%$ of patients treated conservatively died. It's interesting that aneurysm ruptures were observed practically with similar frequency in those operated and non-operated $(33.3 \%$ vs. $31 \%)$. The same trend had been previously registered when we were analyzing 2-, 4- and 6-year treatment period of such patients [26] [27]. There were no operation deaths in patients with DA although almost half of them (45.5\%) had been operated. Two patients died of aortic rupture: 1 operated and 1 non-operated.

After 7 years of treatment, 57.3\% individuals with DAA (68.4\% operated and $54 \%$ non-operated) and $77.3 \%$ with DA ( $90 \%$ operated and $66.7 \%$ non-operated) survived.

Figure 1 shows the main causes of death at 7 years of DAA treatment. It depicts a clearly low death rate due to aneurysm rupture. The main cause of fatal outcomes includes comorbidity and operative mortality and aneurysm ruptures in operated patients who underwent surgery to prevent this in the first place (Figure 1).

\section{Discussions}

Our patients represent primarily (two thirds) a cohort, which can't be urgently or electively operated due to a difficult clinical condition caused by concomitant disease or older age. The remaining third were cases with minor aneurysms, but the age older than 65 (still with comorbidities) was a predominant factor. 
Table 1. Comparison of basic characteristics of DAA after 7 years of sparing treatment.

\begin{tabular}{|c|c|c|c|c|c|c|}
\hline & \multicolumn{3}{|c|}{ Atherosclerotic lesion } & \multicolumn{3}{|c|}{ Dissecting aneurysms } \\
\hline Characteristic & Operated & $\begin{array}{c}\text { Treated } \\
\text { conventionally }\end{array}$ & Total & Operated & $\begin{array}{c}\text { Treated } \\
\text { conventionally }\end{array}$ & Total \\
\hline No. of patients & $19 \%-23.2 \%$ & $63 \%-76.8 \%$ & 82 & $10 \%-45.5 \%$ & $12 \%-54.5 \%$ & 22 \\
\hline $\begin{array}{l}\text { Mortality } \\
\text { by groups }\end{array}$ & $6 \%-31.6 \%$ & $29 \%-46 \%$ & $35 \%-43.9 \%$ & $1 \%-10 \%$ & $4 \%-33.3 \%$ & $5 \%-22.7 \%$ \\
\hline \multicolumn{7}{|l|}{$\begin{array}{l}\text { Including } \\
\text { due to }\end{array}$} \\
\hline Aortic rupture & $2 \%-33 / 3 \%$ & $9 \%-31 \%$ & $11 \%-31.4 \%$ & $1 \%-100 \%$ & $1 \%-25 \%$ & $2 \%-40 \%$ \\
\hline Comorbidity & $1 \%-16.7 \%$ & $20 \%-69 \%$ & $21 \%-60 \%$ & 0 & $3 \%-75 \%$ & $3 \%-60 \%$ \\
\hline Operation & $3 \%-50 \%$ & - & $3 \%-8.6 \%$ & 0 & - & 0 \\
\hline Survivors & $13 \%-68.4 \%$ & $34 \%-54 \%$ & $47 \%-57.3 \%$ & $9 \%-90 \%$ & $8 \%-66.7 \%$ & $17 \%-77.3 \%$ \\
\hline
\end{tabular}

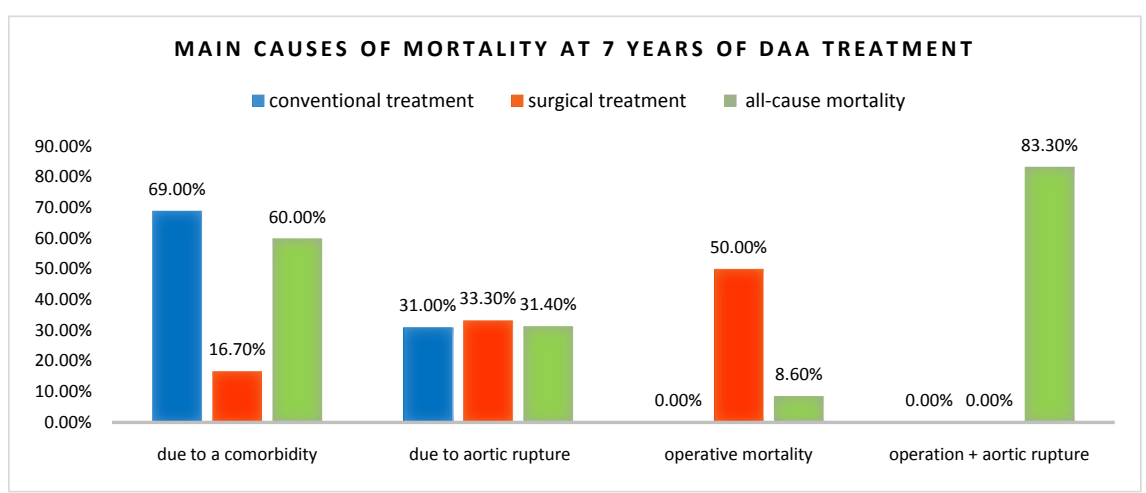

Figure 1. Main causes of mortality at 7 years of DAA treatment.

Despite the younger age, patients with DA couldn't escape mortality due to existing comorbidity. It was $60 \%$ in each group with DAA and DA. Still, no deaths were registered in operated patients with dissecting aneurysm, i.e. the youngest individuals. Among the oldest who received conservative treatment based on their clinical condition, the mortality rate was $69 \%$ in DAA, and $75 \%$ in DA of all deaths between the groups. It suggests that age and concomitant diseases are key factors contributing to a fatal outcome. Some authors say that the presence of concomitant conditions increases to $32 \%-60 \%$ the already great mortality rate after surgical treatment.

Aortic rupture is another factor of death. However, in this case, the death rate is 2 or 3 times lower compared to that observed in the presence of a concomitant disease. Moreover, the percentage of deaths in DAA was similar to that observed in operated and non-operated patients. Similarly, in the group with DA, which contained about the same number of operated and non-operated patients, one patient in each group died. The same trends were registered in our previous analyses of 2, 4 and 5 to 6 years of treatment [26] [27]. The EVAR-2 trial has shown that the cumulated number of deaths due to aneurysm ruptures in those treated conservatively was consistent with the number of ruptures and 
post-operative mortality rate in those receiving endovascular treatments. These trials demonstrate their mismatch with the current approach used to treat DAA.

Based on all these findings, we wonder whether to operate if aneurysm ruptures occur with a similar frequency among operated and non-operated subjects. It seems even more doubtful if to add up operative mortality. Thus, 6 operated patients with DAA died, with 3 of them due to an operation which is $50 \%$; and two due to aortic rupture, which is $33.3 \%$ reaching the factor of $83.3 \%$. Let's compare this value with $69 \%$ deaths due to comorbidities in non-operated subjects. It doesn't favor operative treatment modality.

In operated individuals with DA, neither operative mortality, nor comorbidity-related fatal outcomes were registered suggesting favorable dynamics of this life-threatening disease.

\section{Conclusions}

The pathogenesis of AAA and AAA-related complications is exhibited by two mechanisms which include an increased burden on vascular wall and decreased resistance thereof to such burdens primarily due to the degraded media and inflammatory process involved. Current views link the genesis of structural and functional failure of the aorta with degenerative processes secondary to atherosclerosis and/or activity of intramural proteolytic enzymes, metalloproteases.

DAA and DA share multifaceted etiology and pathogenesis, preventing from thinking that the operative treatment might play a key role in the correction of those conditions. This assumption is based on many years' experience of sparing treatment of our patients. We can see that the dynamics remain unchanged despite ever-changing measurements of the parameters. It is noteworthy, that the frequency of aneurysm ruptures is not always affected by operative treatment, including DAs.

Medical treatment seems to be more often than not consistent with etiopathogenesis of the disease compared to active surgical modality. Therefore, we opt for medical therapy even after surgery to stabilize the condition. The survival rates confirm the feasibility of this approach. In recent years, different survival rates have been mentioned in various data based on the diameter of the aneurysm. In the presence of minor abdominal aneurysms, survival was $48 \%$ at 5 years. If it was more than $6 \mathrm{~cm}$, survival was $50 \%$ at 1 year spiraling down to just $6 \%$ at 5 years [1] [28] [29] [30]. Russian national guidelines (2010) describe English data on survival from DAA in the period between 6 to 10 years. It was $57 \%$ in operated patients and $52 \%$ in the study group.

When sparing treatment modality was used to treat DAA, the survival rate reached $90.1 \%$ at 2 years, $76.8 \%$ at 4 years, $59.4 \%$ at 6 years, and $57.5 \%$ at 7 years with uniform increase in mortality primarily due to a concomitant condition. Survival in the group with DA (77.3\%) was better due to the younger age of patients. It was $68.4 \%$ in operating due to DAA at 7 years, and $54 \%$ in non-operated patients. 


\section{Conflicts of Interest}

The authors declare no conflicts of interest regarding the publication of this paper.

\section{References}

[1] Shirinbek, O. (2008) Infrarenal Abdominal Aneurisms: Modalities and Outcomes (Literature Review). Cardiovascular Disease Journal, 9, 50-57. (In Russian)

[2] Dua, M.M. and Dalman, R.L. (2010) Hemodynamic Influences on Abdominal Aortic Aneurism Disease. Application of Biomechanics to Aneurysm Pathophysiology. Vascular Pharmacology, 53, 11-21. https://doi.org/10.1016/j.vph.2010.03.004

[3] Aggarwal, S., Qamar, A., Sharma, V. and Sharma, A. (2011) Abdominal Aortic Aneurysm: A Comprehensive Review. Experimental \& Clinical Cardiology, 16, 11-15.

[4] Fleming, C., Whitlock, E.P., Beil, T.L., et al. (2005) Screening for Abdominal Aortic Aneurysm: A Best-Evidence Systematic Review for the US Preventive Services Task Force. Annals of Internal Medicine, 142, 203-211. https://doi.org/10.7326/0003-4819-142-3-200502010-00012

[5] John, K.A., Thomas, L.F. and Serrius Patrick, V. (2011) Cardiovascular Disease Edition. “ESC Guidelines” GEOTAR-Media, 1209-1248. (In Russian)

[6] Capri, M., Salvioli, S., Sevini, F., et al. (2006) The Genetics of Human Longevity. Annals of the New York Academy of Sciences, 1067, 252-263. https://doi.org/10.1196/annals.1354.033

[7] Wang, M., Zhang, J., Jiang, L.Q., et al. (2007) Pro-Inflammatory Profile within the Grossly Normal Aged Human Aortic Wall. Hypertension, 50, 219-227. https://doi.org/10.1161/HYPERTENSIONAHA.107.089409

[8] Venugopal, S.K., Devaraj, S., Yuhanna, I., Shaul, P. and Jialal, I. (2002) Demonstration that C-Reactive Protein Decreases eNOS Expression and Bioactivity in Human Aortic Endothelial Cells. Circulation, 106, 1439-1441. https://doi.org/10.1161/01.CIR.0000033116.22237.F9

[9] Wang, M., Zhang, J., Spinetti, G., et al. (2005) Angiotensin II Activates Matrix Metalloproteinase Type II and Mimics Age-Associated Carotid Arterial Remodeling in Young Rats. The American Journal of Pathology, 167, 1429-1442. https://doi.org/10.1016/S0002-9440(10)61229-1

[10] Lim, M.A. and Townsend, R.R. (2009) Arterial Compliance in the Elderly: Its Effect on Blood Pressure Measurement and Cardiovascular Outcomes. Clinics in Geriatric Medicine, 25, 191-205. https://doi.org/10.1016/j.cger.2009.01.001

[11] Boersma, E., Kertai, M.D., Schouten, O., et al. (2005) Perioperative Cardiovascular Mortality in Non-Cardiac Surgery: Validation of the Lee Cardiac Risk Index. The American Journal of Medicine, 118, 1134-1141. https://doi.org/10.1016/j.amjmed.2005.01.064

[12] Lederle, F.A., Wilson, S.E., Johnson, G.R., et al. (2002) Aneurysm Detection and Management Veterans Affairs Cooperative Study Group. Immediate Repair Compared with Surveillance of Small Abdominal Aortic Aneurysm. The New England Journal of Medicine, 346, 1437-1444.

[13] Williams, B., Lacy, P.S., Thom, S.M., et al. (2006) for the CAFÉ investigators. Differential Impact of Blood Pressure-Lowering Drugs on Central Aortic Pressure and Clinical Outcomes. Principal Results of the Conduit Artery Function Evaluation (CAFÉ) Study. Circulation, 113, 1213-1225.

https://doi.org/10.1161/CIRCULATIONAHA.105.595496 
[14] Prozorovskaya, N.N., Glinyanaya, S.V., Gerastchenko, L.P., et al. (1988) Impact of Beta-Blocker Therapy and Vitamin Supplements on Oxyproline Secretion in the Context of Heritable Connective Tissue Disorders. Voprosi Meditsinskoy Khimii, 34, 99-104. (In Russian)

[15] Sudakov, K.V. (2005) Individual Nature of Emotional Stress. Nevrologiya i psikhiatriya, 5, 5-10. (In Russian)

[16] Ceconi, C., Francolini, G., Bastianon, D., et al. (2007) Differences in the Effect of Angiotensin-Converting Enzyme Inhibitors on the Rate of Endothelial Cell Apoptosis: In Vitro and in Vivo Studies. Cardiovascular Drugs and Therapy, 21, 423-429. https://doi.org/10.1007/s10557-007-6068-5

[17] Virdis, A., Ghiadoni, L. and Taddei, S. (2011) Effects of Antihypertensive Treatment on Endothelial Function. Current Hypertension Reports, 13, 276-281. https://doi.org/10.1007/s11906-011-0207-x

[18] Manisty, C.H., Zambanini, A., Parker, K.H., et al. (2009) Differences In the Magnitude of Wave Reflection Account for Differential Effects of Amlodipine-Versus Atenolol-Based Regimens on Central Blood Pressure: An Anglo-Scandinavian Cardiac Outcome Trial Sub-Study. Hypertension, 54, 724-730. https://doi.org/10.1161/HYPERTENSIONAHA.108.125740

[19] Rothwell, P.M., Howard, S.C., Dolan, E., et al. (2010) Effects of Beta-Blockers and Calcium Channel Blockers on within-Individual Variability in Blood Pressure and Risk of Stroke. The Lancet Neurology, 9, 469-480. https://doi.org/10.1016/S1474-4422(10)70066-1

[20] Vammen, S., Lindholt, J.S., Østergaard, L., Fasting, H. and Henneberg, E.W. (2001) Randomized Double Blind Controlled Trial of Roxithromycin for Prevention of Abdominal Aortic Aneurysm Expansion. British Journal of Surgery, 88, 1066-1072. https://doi.org/10.1046/j.0007-1323.2001.01845.x

[21] Chernomortseva, E.S., Pokrovsky, M.V., Titaryova, L.V., et al. (2010) A Multifaceted Assessment of Endothelial Protector and Cardioprotector Activity of Macrolide Antimicrobials to Model Nitric Oxide Deficiency. Eksperimentalnaya i Klinicheskaya Farmakologiya, 9, 20-23.

[22] Ejiri, J., Jnoue, N., Tsucube, T., et al. (2003) Oxidative Stress in Pathogenesis of Thoracic Aortic Aneurysm: Protective Role of Statin And Angiotensin II Type I Receptor Blocker. Cardiovascular Research, 59, 988-996.

https://doi.org/10.1016/S0008-6363(03)00523-6

[23] Mrochek, A.G. and Gubar, E.N. (2007) Hypercholesterolemia, Statins and the Role of Atorvastatin (LipiMAX ${ }^{\circledast}$ ) To Treat Atherosclerosis and Its Complications. Ars Medica, 1, 33-41. (In Russian)

[24] Taneva, E., Borucki, K. and Wiens, L. (2006) Early Effects on Endothelial Function of Atorvastatin $40 \mathrm{mg}$ Twice Daily and Its Withdrawal. American Journal of Cardiology, 97, 1002-1006. https://doi.org/10.1016/j.amjcard.2005.10.032

[25] Arakelyan, V.S. and Zhane, A.K. (2012) Coronary Artery Lesion in Patients with Infrarenal Abdominal Aneurysm. Kreativnaya Kardiologiya, 2, 56-66. (In Russian)

[26] Krylov, V.P., Manak, N.A., Gayduk, V.N., Mankevich, N.V. and Reut, L.I. (2017) Ways to Correct Indications for Surgical Treatment of Atherosclerotic Descending Aortic Aneurysms. Advances in Surgical Sciences, 5, 31-35. https://doi.org/10.11648/j.ass.20170503.11

[27] Krylov, V.P., Shevtsov, D.E., Popel, G.A., Zhigalkovich, A.S., et al. (2018) Sparing Treatment of Thoracic and Abdominal Aortic Aneurysms. Health, 10, 458-466. https://doi.org/10.4236/health.2018.104037 
[28] Schepens, M.A., Kelder, J.C., Morshuis, W.J., et al. (2007) Long-Term Follow-Up after Toracoabdominal Aortic Aneurysm Repair. The Annals of Thoracic Surgery, 83, S851-S855. https://doi.org/10.1016/j.athoracsur.2006.10.087

[29] Sweeting, M.J., Thompson, S.G., Brown, L.C., Greenhalgh, R.M. and Powell, J.T. (2010) Use of Angiotensin Converting Enzyme Inhibitors Is Associated with Increased Growth Rate of Abdominal Aortic Aneurysms. Journal of Vascular Surgery, 52, 1-4. https://doi.org/10.1016/j.jvs.2010.02.264

[30] Sweeting, M.J., et al. (2012) Meta-Analysis of Individual Patient Data to Examine Factors Affecting Growth and Rupture of Small Abdominal Aortic Aneurysms. British Journal of Surgery, 99, 655-665. https://doi.org/10.1002/bjs.8707 